\title{
Abnormal levels of age-elastin derived peptides in sera of diabetic patients with arterial hypertension
}

\author{
ASPARUH NIKOLOV ${ }^{1}$, IVAN TSINLIKOVI, GEORGE NICOLOFF ${ }^{2}$, IVANKA TSINLIKOVA ${ }^{l}$, \\ ALEXANDER BLAZHEV $V^{2}$, ANTOAN GAREV $V^{l}$
}

'Department of Propedeutics of Internal Diseases, Medical University, Pleven, Bulgaria

${ }^{2}$ Division of Biology and Immunology, Medical University, Pleven, Bulgaria

\begin{abstract}
Introduction: An important factor in vascular wall alterations is degradation of elastic fiber major protein - elastin. As a result, elastin derived peptides (EDP) are found in circulation. Advanced glycation might also involve elastin, because it is a protein with slow metabolism. The aim of our study was to measure serum levels of glycated elastin derived peptides (AGE-EDP) of elastin in patients with type 2 diabetes mellitus (T2DM) and arterial hypertension (AH).

Material and methods: We adapted an ELISA technique for the determination of AGE-EDP. Sera of 93 patients with T2DM and AH (mean age $61.4 \pm 11.3$ years, diabetes duration $9.88 \pm 3.12$ years; hypertension duration $9.28 \pm 4.98)$ were tested. These values were compared to 42 age-and sex-matched controls. Diabetics were divided in two groups according to presence - Group $1(n=67)$ or absence Group $2(n=26)$ of microangiopathy.

Results: Patients with T2DM and AH showed statistically significantly higher levels of AGEEDP in comparison with healthy controls $0.060(0.053 \div 0.065)$ vs. $0.039(0.031 \div 0.044)(K W=35.2$; $p<0.0001)$. Group 1 showed significantly higher levels of AGE-EDP than the control group 0.069 $(0.051 \div 0.070)$ vs. $0.039(0.031 \div 0.044)(K W=33.0 ; p<0.0001)$. Group 2 also showed significantly higher levels of $A G E-E D P$ than controls $0.058(0.049 \div 0.064)$ vs. $0.039(0.031 \div 0.044)(K W=22.1$; $p<0.0001)$. AGE-EDP showed a correlation with an insulin dose $(r=-0.28 ; p=0.05)$, systolic blood pressure $(r=0.25 ; p=0.01), B M I(r=0.39 ; p=0.01)$ and retinopathy $(r=0.18 ; p=0.05)$.

Conclusions: The measurement of non-invasive markers of elastin glycation may be useful in monitoring development of vascular wall alterations and therapeutic interventions.
\end{abstract}

Key words: ELISA, elastin, AGEs, diabetic microvascular complications, arterial hypertension.

(Centr Eur J Immunol 2014; 39 (3): 345-351)

\section{Introduction}

Patients with type 2 diabetes mellitus (T2DM) are at a high risk of development of vascular disease. This risk is increased with existence of arterial hypertension. In diabetic patients, morbidity and mortality are mainly related to the presence of late complications, namely macro-and microangiopathy. Advanced glycation end-products (AGEs) are generated in diabetes mellitus as a result of chronic hyperglycemia and enhanced oxidative stress. These AGEs, via direct and receptor-dependent pathways, promote the development and progression of cardiovascular disease.

Advanced glycation end-products of non-enzymingglycation/non-enzymatic glycation are a heterogeneous group of chemical components, which are formed through non-enzymingglycation, i.e. through connection to reduced glucose molecule (also known as glycooxygenation or glycosylation) to proteins, fats and nucleid acids. The term "final product" has been chosen because AGEs are the final product of chain reactions generating some osculant products (for example Schiff bases, Amadori products, Maillard products, deoxyglucosone, methylglyoxal glycolaldehyde). Most important AGEs include carboxymethyl lysine (CML), carboxyethyl lysine (CEL), pentosidine and hydroimidazolone. Carboxymethyl lysine is known as "AGEs-indicator" [1]. Advanced glycation end-products and their receptors, RAGE, can be detected in nearly each body cell and their major role has been discovered in progress of atherosclerosis and particularly in patients with diabetes. In the serum of patients with diabetes type II, significantly higher concentrations of AGEs compared to

Correspondence: Asparuh Nikolov, Department of Propedeutics of Internal Diseases, Medical University, Pleven, Bulgaria, e-mail: a_nicoloff@yahoo.com 
healthy people are seen and this is related with a common higher cardiovascular mortality rate. Accumulation of AGEs is related with development and progression of late injury of diabetes independently of glu- $\mathrm{HbA}_{1 \mathrm{c}}$ levels, so in this way metabolic stress rises [2].

Numerous studies have investigated the role of the AGEs in diabetic subjects; however, the role in hypertension and the cardiovascular system has been less intensively investigated in clinical studies. Clinical data on the AGE-RAGE axis at the moment does not provide evidence for a role in hypertension but for vascular disease, including macro circulation as well as microcirculation [3].

Advanced glycation end-products play an important role in the pathogenesis of vascular diseases via deposition in the arterial wall. There is evidence of accelerated AGE formation in hypertension and plasma levels of AGEs are significantly higher in hypertensive patients compared with normotensive subjects [3]. The formation of advanced glycation end-products is associated with arterial stiffness in experimental models and alagebrium (formerly known as ALT-711), an advanced glycation end-product cross-link breaker, has been shown to reduce arterial stiffness in elderly subjects. McNulty et al. [4] related plasma concentrations of AGEs, measuring a noncompetitive immunoassay, and markers of aortic stiffness-pulse wave velocity (PWV) and augmentation index (AIx), a measure of aortic wave reflection - in 46 subjects, aged $47 \pm 2$ years, comprising 30 untreated hypertensive and 16 normotensive subjects. Plasma AGEs were significantly higher in hypertensive than in normotensive subjects $(7.8 \pm 1$ vs. $3 \pm 1 \mathrm{mug} / \mathrm{ml}$; $p<0.0001)$. There was a significant relationship between plasma AGEs and aortic PWV $(r=0.49, p<0.01)$, but not with AIx. In a stepwise regression model age, plasma AGE levels, smoking status, and total cholesterol explained $67 \%$ of the variability in PWV. For AIx, the only variables that entered the model were age, gender, and heart rate $\left(r^{2}=\right.$ $=0.53, p<0.0001)$ with no contribution from plasma AGEs. Authors conclude that a concentration of plasma AGEs is significantly higher in hypertensive than in normotensive subjects and related to aortic stiffness independent of age and blood pressure, with no relationship with aortic wave reflection. Plasma AGEs may play a blood pressure-independent role in large but not small vessel remodeling in essential hypertension.

Advanced glycation end-product formation affects the physiological properties of proteins in extracellular matrix, such as turnover and elasticity [2]. Hartog et al. [5] investigated the relationship between AGEs and diastolic function and the response to blood pressure treatment in patients with hypertension and diastolic dysfunction. Data were analysed from 97 patients (aged $65 \pm 10$ years, 36\% male) who were randomly assigned to 6 months' open-label treatment with either eprosartan on top of other anti-hypertensive drugs $(n=47)$ or other anti-hypertensive drugs alone $(n=50)$. Tissue AGE accumulation was mea- sured using a validated skin-autofluorescence (skin-AF) reader $(n=26)$. Plasma $\mathrm{N}$ (epsilon)-(carboxymethyl)lysine (CML), N(epsilon)-(carboxyethyl)lysine (CEL), and pentosidine were measured by LC-MS/MS and HPLC. Diastolic function was assessed using echocardiography. Blood pressure was reduced from 157/91 to $145 / 84 \mathrm{~mm} \mathrm{Hg}$ $(p<0.001)$ in the eprosartan group and from 158/91 to $141 / 83 \mathrm{~mm} \mathrm{Hg}(p<0.001)$ in the control group. No effect of eprosartan was found on AGE levels. In patients with baseline skin-AF $<$ median, E/A ratio $(p=0.04)$ and the mean peak early-diastolic filling velocity (E') improved $(p=0.001)$. In contrast, in patients with skin-AF levels $>$ median, E/A ratio $(p=0.84)$ and mean E' $(p=0.32)$ remained unchanged. Although eprosartan did not decrease levels of AGEs, patients with lower skin-AF at baseline showed a larger improvement in diastolic function in response to either anti-hypertensive treatment compared with patients with higher skin-AF. Authors conclude that hypertension is related to a higher risk of development of heart failure.

An increase in the amount of advanced glycation end-products (AGE) is seen in the blood of patients with diabetes mellitus $[6,7]$. This increase is believed to play a causal role in diabetic neuropathy [8], nephropathy [6,9] and retinopathy [10-13]. Glucose reacts non-enzymatically with proteins to form Schiff base and Amadori products, which are early stage products. Further incubation of early stage products leads to the formation of AGE [14].

The extracellular matrix protein elastin is responsible for the major part of tissue elasticity and is an insoluble component of elastic fibers in skin, lung and arteries [15]. The detection of circulating elastin-derived peptides (EDP) in the serum of healthy subjects $[16,17]$ shows that the elastin macromolecule is not fully degraded by elastases in vivo. These EDP may become a stimulus for immunocompetent cells and result in the production of anti-elastin antibodies (AEAbs) [18]; these can also be detected in the serum of healthy human subjects [19-21]. We formulated a hypothesis that AEAbs may play a physiological role in elastin turnover [19]. This role may be either: (i) to enhance the final elimination of EDP from the serum; (ii) to mark the altered elastic structures and block the chemotactic activity of EDP for elastase-producing cells [22] and thus to regulate the activity of the elastolytic process; or (iii) AEAbs may bind EDP and block the recognition of the EDP by the immunocompetent cells and prevent their binding to the elastin receptor. Hence the activity of the immune response would be restricted to elastin.

The aim of the present study was to try to measure serum AGE-EDP concentrations in healthy and diabetic patients with hypertension. For that purpose, 93 patients with T2DM and arterial hypertension (AH) (mean age 61.4 \pm 11.3 years, diabetes duration $9.88 \pm 3.12$ years, hypertension duration $9.28 \pm 4.98$ ) were tested. These values were compared to serum AGE-EDP levels in 42 age- and sex- 
matched controls. Diabetics were divided in two groups according to presence - Group $1(n=67)$ or absence Group $2(n=26)$ of microangiopathy.

\section{Material and methods}

\section{Subjects}

The experimental group consisted of 93 patients (37 men, 56 women) with T2DM and AH (mean age 61.4 \pm 11.3 years, diabetes duration $9.88 \pm 3.12$ years, hypertension duration $9.28 \pm 4.98$ ). These values were compared to serum antibodies to elastin in 42 age-and sex-matched controls with no family history of diabetes, atherosclerosis or emphysema. The controls were equally distributed to match the diabetic ages. All patients signed informed consent prior to the study start.

Diabetics were divided in two groups according to presence - Group $1(n=67)$ or absence - Group $2(n=26)$ of microvascular complications (Table 1). Group 1 consisted of $39 \%$ of men and $61 \%$ of women. Fifty-five percent were smokers and $45 \%$ non-smokers. Group 2 consisted of $42 \%$ of men and $58 \%$ of women. Fifty-eight percent were smokers and $42 \%$ non-smokers. Controls consisted of $45 \%$ of men and $55 \%$ of women. Twenty-seven percent were smokers and $73 \%$ non-smokers (Table 2). Microalbuminuria was defined as a persistent urinary albumin excretion rate (AER) in the range of 20 and $200 \mu \mathrm{g} / \mathrm{min}$ in sterile urine. None of the patients had a diagnosis of renal disease unrelated to diabetes during the follow-up.

\section{Antigen preparation}

AGE-elastin was obtained via incubation of human aortic $\alpha$-elastin $\left(1.33 \mathrm{mg} / \mathrm{ml}^{-1}\right)$ with $100 \mathrm{mmol} / \mathrm{l}^{-1}$ glucose, in $0.2 \mathrm{M}$ phosphate buffer, $\mathrm{pH} 7.8$, containing $0.04 \%$ sodium azide, at $37^{\circ} \mathrm{C}$ for 30 days as described by Baydanoff et al. [16].

\section{Production of immune serum}

Polyclonal immune serum against AGE of Hemocyanin from Keyhole Limpets (AGE-KLH) was produced in rabbit as described by Baydanoff et al. [16].

Polyclonal immune serum against human aortic $\alpha$-elastin was produced in sheep as described by Baydanoff et al. [16].

\section{Measurement of AGE-EDP in human sera}

An ELISA was used for detection of AGE-EDP. The wells of polystyrene plates were coated with $10 \mu \mathrm{g} / \mathrm{ml}$ of rabbit anti-AGE-KLH IgG in $0.05 \mathrm{M}$ sodium carbonate buffer $\left(\mathrm{pH}\right.$ 9.6) and were incubated for $3 \mathrm{~h}$ at $37^{\circ} \mathrm{C}$ and overnight at $4 \mathrm{C}$. After washing three times with PBS containing $0.01 \%$ Tween 20 , the wells were incubated with PBS containing $0.02 \% \mathrm{NaN}_{3}$ and $0.5 \%$ bovine serum albumin (BSA) for $1 \mathrm{~h}$ at $37^{\circ} \mathrm{C}$ to block unspecific binding. After washing wells 3 times, $100 \mu$ l of human sera (diluted $1: 5$ ) were placed in each well. Incubation time was $1 \mathrm{~h}$ at $37^{\circ} \mathrm{C}$. The plates were washed 3 times and the wells were incubated with $10 \mu \mathrm{g} / \mathrm{ml}$ of sheep anti-elastin IgG for $1 \mathrm{~h}$ at $37^{\circ} \mathrm{C}$. The plates were then washed and donkey anti-sheep IgG peroxidase immunoconjugate (SIGMA, USA) diluted 1: 10000 was used for $1 \mathrm{~h}$ incubation at $37^{\circ} \mathrm{C}$. After washing 3 times, o-Phenylenediamine $(0.4 \mathrm{mg} / \mathrm{ml})$ was added to citrate buffer, and $100 \mu$ of this solution was added to each well and allowed to react for $30 \mathrm{~min}$. The reaction was stopped by the addition of $50 \mu \mathrm{l}$ of $4 \mathrm{M} \mathrm{H}_{2} \mathrm{SO}_{4}$ and absorbance was read on a microplate reader. A standard curve using AGE-elastin was obtained in the range from $1.25 \mathrm{ng} / \mathrm{ml}$ to $10 \mu \mathrm{g} / \mathrm{ml}$.

\section{Controls}

Three types of negative controls confirmed the immunospecificity of antibodies:

- substituting sheep non-immune IgG for the primary antibody,

- substituting rabbit IgG for the secondary antibody,

- omitting the primary antibody in the procedure,

- omitting the secondary antibody in the procedure,

- substituting a human sample with $\alpha$-elastin,

- substituting a human sample with AGE-KLH.

One type of positive control was used:

- substituting a human sample with AGE- $\alpha$-elastin.

\section{Characterization of immune antibodies}

The anti-AGE antibodies reacted with AGE epitopes regardless of the protein. For example our antibodies recognize AGE-elastin, AGE-BSA, and AGE-KLH in ELISA (Baydanoff et al. 1996).

\section{Other methods}

Ophthalmoscopy through dilated pupils was carried out in all diabetic patients to assess the presence of retinopathy, with all patients studied by the same ophthalmologist. Glycated hemoglobin, serum total cholesterol and triglyceride concentrations, arterial blood pressure and AER were examined as described by Nicoloff et al. [18].

\section{Statistical analyses}

The research data were processed with the computer programs EXCEL (Microsoft Corporation, Redmond, WA) and STATGRAPHICS plus (Manugistics, Rockville, MD) for WINDOWS. For assessment and conclusions in the case of normal distribution, values are expressed as mean $(\mathrm{x}) \pm$ standard deviation (SD). The Student's $t$-test, $\mathrm{F}$ ratio, a one-way analysis of variance (ANOVA) were used to assess differences between study groups for normal distribution (Least significant difference, Tukey honest significant difference, Scheffe', Bonferroni, Newman-Keuls, and Duncan tests). Kruskal-Wallis test was 
Table 1. Clinical data of patients with type 2 diabetes mellitus and arterial hypertension

\begin{tabular}{|c|c|c|c|}
\hline Clinical data & Group 1 & Group 2 & Controls \\
\hline age & $62.5 \pm 12.58$ & $60.4 \pm 8.4$ & $58.9 \pm 7.56$ \\
\hline sex (male/female) & $26 / 41$ & $11 / 15$ & $20 / 22$ \\
\hline average duration of diabetes & $9.30 \pm 5.36$ & $9.16 \pm 7.59$ & N/A \\
\hline average duration of hypertension & $9.50 \pm 7.63$ & $8.68 \pm 7.26$ & N/A \\
\hline $\mathrm{HbA}_{1 \mathrm{c}}$ & $7.63 \pm 2.03^{*}$ & $7.27 \pm 1.63$ & N/A \\
\hline systolic $\mathrm{AH}(\mathrm{mm} \mathrm{Hg})$ & $142.83 \pm 18.05$ & $140.58 \pm 20.51$ & $114.29 \pm 15.74$ \\
\hline diastolic $\mathrm{AH}(\mathrm{mm} \mathrm{Hg})$ & $82.23 \pm 11.52$ & $81.35 \pm 11.96$ & $72.5 \pm 10.4$ \\
\hline BMI & $29.62 \pm 4.99$ & $28.42 \pm 3.96$ & $22.61 \pm 2.27$ \\
\hline common cholesterol (mmol/l) & $5.26 \pm 1.40^{*}$ & $5.18 \pm 0.93$ & $3.99 \pm 0.65$ \\
\hline HDL (mmol/l) & $0.88 \pm 0.30^{*}$ & $0.93 \pm 0.30$ & $0.96 \pm 0.20$ \\
\hline LDL (mmol/l) & $3.18 \pm 1.19$ & $3.16 \pm 1.09$ & $2.43 \pm 0.64$ \\
\hline triglycerides $(\mathrm{mmol} / \mathrm{l})$ & $2.91 \pm 1.68$ & $2.53 \pm 1.49$ & $1.31 \pm 0.61$ \\
\hline insulin dose $(\mathrm{U} / \mathrm{kg} / 24 \mathrm{~h})$ & $2.57 \pm 0.52$ & $2.03 \pm 0.93$ & N/A \\
\hline MAU $(\mu \mathrm{g} / \mathrm{min})$ & $78.94 \pm 52.87^{*}$ & $8.53 \pm 4.69$ & N/A \\
\hline MAU & $(n=43)$ & - & \\
\hline retinopathy & $(n=20)$ & - & \\
\hline neuropathy & $(n=4)$ & - & \\
\hline smokers & $37 / 67$ & $15 / 26$ & $16 / 42$ \\
\hline number of patients & 67 & 26 & 42 \\
\hline
\end{tabular}

N/A - not aberrant, Values are Mean \pm SD.

Group 1 - patients with vascular complications $(n=67)$, Group 2 - patients without vascular complications $(n=26)$, Controls $(n=42)$

Group 1 (patients with microvascular complications) show higher mean values of HbAlc, total cholesterol, and microalbuminuria than patients from Group 2.

Table 2. Distribution (in per cent) of patients in tvhe groups according to sex and presence or absence of smoking as a factor

\begin{tabular}{ccccc}
\hline & \multicolumn{2}{c}{ Sex } & \multicolumn{2}{c}{ Smoking } \\
\cline { 2 - 5 } & male & female & smokers & non-smokers \\
\hline Group 1 & $39 \%$ & $61 \%$ & $55 \%$ & $45 \%$ \\
\hline Group 2 & $42 \%$ & $58 \%$ & $58 \%$ & $42 \%$ \\
\hline Controls & $45 \%$ & $55 \%$ & $47 \%$ & $53 \%$ \\
\hline
\end{tabular}

Group 1 consisted of 39\% of men and 61\% of women. Fifty-five percent were smokers and $45 \%$ non-smokers. Group 2 consisted of $42 \%$ of men and $58 \%$ of women. Fifty-eight percent were smokers and $42 \%$ non-smokers. Controls consisted of $45 \%$ of men and $55 \%$ of women. Twenty-seven percent were smokers and $73 \%$ non-smokers.

used to compare the medians for non-normal distribution. In cases with non-normal distribution, median value was used (M), together with the first and third quartile Q1 and Q3 (twenty-fifth and seventy-fifth percentile P25 and 75P). For selected data sets, a correlation analysis was performed and data were considered significant with a $p$ value of less than 0.05 .

\section{Results}

None of the negative controls of ELISA showed a positive reaction.
Patients with T2DM and AH showed statistically significantly higher levels of AGE-EDP in comparison with healthy controls $0.060(0.053 \div 0.065)$ vs. $0.039(0.031$ $\div 0.044)(\mathrm{KW}=35.2 ; p<0.0001)$ (Fig. 1 , Table 3$)$. Group 1 showed significantly higher levels of AGE-EDP than the control group $0.069(0.051 \div 0.070)$ vs. $0.039(0.031 \div 0.044)$ $(\mathrm{KW}=33.0 ; p<0.0001)$. Group 2 also showed significantly higher levels of AGE-EDP than controls 0.058 (0.049 $\div 0.064)$ vs. $0.039(0.031 \div 0.044)(\mathrm{KW}=22.1 ; p<0.0001)$. The highest serum levels of glycated elastin were found in patients with microvascular complications. AGE-EDP showed a correlation with the insulin dose $(r=-0.28$; 
$p=0.05)$, systolic blood pressure $(r=0.25 ; p=0.01)$, BMI $(r=0.39 ; p=0.01)$ and retinopathy $(r=0.18 ; p=0.05)$.

\section{Discussion}

Oxidative stress activated by AGEs leads to tissue dysfunction, atherosclerosis plaque and in the end myocardial infarction and insult. Because of that, it is so hard to prevent diabetes injury despite good controlled blood-sugar balance [23].

It was shown that vascular extracellular matrix and especially elastin fibers are degraded during aging and atherosclerosis [24, 25]. The loss of elasticity and increasing rigidity of the aging arterial wall can be attributed to loss of elastin fiber integrity and stiffening of the collagen fiber network by maintained or increased synthesis and crosslinking by the Maillard reaction [26]. The crosslinking as a result of the Maillard reaction, consisting in the formation and progressive modification of the structure of glucose-amine addition products (aldol condensation, Schiff base formation) resulting in the production of AGE. Some of them are efficient in crosslinking proteins and nucleic acids (Ikan 1996). The condensation of glucose or of other reducing sugars or aldehydes with amine groups on proteins or nucleic acids was studied in great detail. There is no known natural defense mechanism against the Maillard reaction. Nature apparently neglected to protect the organisms against these effects of one of the most important metabolites and energy source of the cells [10].

Elastin is an extracellular matrix protein biopolymer, which allows organs such as lungs, skin and blood vessels to stretch repeatedly with no loss of elasticity [27]. Elastin is considered to be a stable tissue constituent with essentially no measurable turnover [28]. There are, however, arguments in favor of its constant partial degradation and also for its neosynthesis. This was observed in the aging vascular wall [24] as well as in skin [28].
In our investigation we measured serum concentrations of glycated elastin, glycated in vitro as an antigen, expressing AGE epitopes, common to all glycated proteins. The reason was that glycation of hemoglobin forms $\mathrm{HbA}_{1 \mathrm{c}}$ that has been described as an Amadori product but is not an AGE [29]. It is an indicator of glycemia from the preceding 6 to 12 weeks, whereas advanced glycation

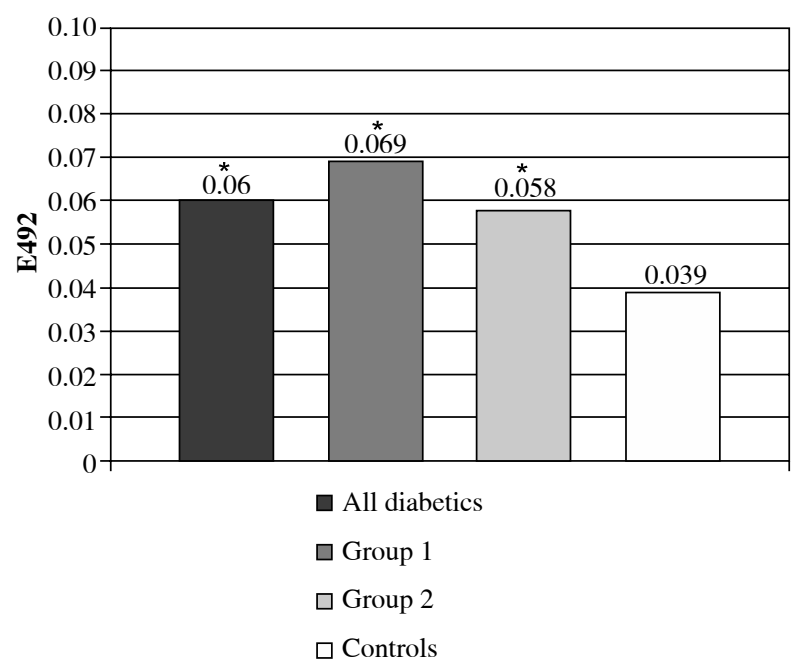

E492 - extinction coefficient

Patients with T2DM and AH showed statistically significantly higher levels of AGE-EDP in comparison with healthy controls $0.060(0.053$ $\div 0.065)$ vs. $0.039(0.031 \div 0.044)(\mathrm{KW}=35.2 ; p<0.0001)$. Group 1 showed significantly higher levels of AGE-EDP than the control group $0.069(0.051 \div 0.070)$ vs. $0.039(0.031 \div 0.044)(\mathrm{KW}=33.0$; $p<0.0001)$. Group 2 also showed significantly higher levels of AGEEDP than controls $0.058(0.049 \div 0.064)$ vs. $0.039(0.031 \div 0.044)(\mathrm{KW}$ $=22.1 ; p<0.0001)$. The highest levels of gly-elastin were found in patients with microvascular complications.

Fig. 1. Levels of serum AGE-EDP in patients with type 2 diabetes mellitus and arterial hypertension

Table 3. Levels of serum AGE-EDP in patients diabetes type 2 and AH

\begin{tabular}{|c|c|c|c|c|}
\hline \multirow[t]{2}{*}{ Groups } & \multirow{2}{*}{$\frac{\text { AGE-EDP }(\mathrm{ng} / \mathrm{ml})}{\mathrm{M} \div(\mathrm{Q} 1-\mathrm{Q3})}$} & \multicolumn{3}{|c|}{ Compared to others } \\
\hline & & group 1 & group 2 & all diabetics \\
\hline all diabetics & $\begin{array}{c}0.060 \\
(0.053 \div 0.065)\end{array}$ & NS & NS & - \\
\hline group 1 & $\begin{array}{c}0.069 \\
(0.051 \div 0.070)\end{array}$ & - & NS & NS \\
\hline group 2 & $\begin{array}{c}0.058 \\
(0.049 \div 0.064)\end{array}$ & NS & - & NS \\
\hline controls & $\begin{array}{c}0.039 \\
(0.031 \div 0.044)\end{array}$ & $* p<0.0001$ & $* p<0.001$ & $* p<0.0001$ \\
\hline
\end{tabular}

$N S$ - non-significant, $\div(Q 1-Q 3)$ - median value, $Q 1$ - first quartile, $Q 3$ - third quartile

Patients with T2DM and AH showed statistically significantly higher levels of AGE-EDP in comparison with healthy controls 0.060 (0.053 $\div 0.065)$ vs. 0.039 $(0.031 \div 0.044)(K W=35.2 ; p<0.0001)$. Group 1 showed significantly higher levels of AGE-EDP than the control group $0.069(0.051 \div 0.070)$ vs. $0.039(0.031$ $\div 0.044)(K W=33.0 ; p<0.0001)$. Group 2 also showed significantly higher levels of AGE-EDP than controls $0.058(0.049 \div 0.064)$ vs. $0.039(0.031 \div 0.044)(K W$ $=22.1 ; p<0.0001)$. 
reflects a process that can occur over a longer period [30]. Many hypotheses for the etiology of arterial disease involve changes in the extracellular matrix and it is possible that the increased susceptibility in diabetes mellitus is associated with glycation, arising from reaction between glucose and the amino groups on proteins. Of the extracellular matrix molecules, elastin has been suitable as a substrate for these reactions because it has the lowest turnover rate and is therefore at risk of modification even by very slow chemical reactions. On the other hand, its mechanical properties are crucially important in normal arterial function and it is widely implicated in disease, either through loss of its mechanical properties or because it acts as a substrate for calcification and lipid deposition.

In this study, using a sandwich version of ELISA we tried to measure the serum concentrations of AGE-EDP among type 2 diabetes patients with arterial hypertension and to test the possible association between AGE-EDP and diabetic vascular complications. Diabetics with vascular complications showed significantly higher concentrations of EDP and AGE-EDP than those without vascular complications. Serum AGE-EDP concentrations correlated with microangiopathy especially retinopathy $(p=0.05)$ and systolic blood pressure $(p=0.01)$, BMI $(p=0.01)$. We interpreted the association of AGE-EDP and hypertension as follows: increased levels of serum glycated elastin may accelerate atherosclerosis and induce development and progression of cardiovascular disease. AGE-EDP can accumulate at many sites of the body predominantly in small blood vessels, which are impaired in both diabetes and hypertension. These biological effects translate to accelerated plaque formation.

Arterial hypertension and diabetes mellitus are both characterized by insulin resistance. In insulin resistance, alterations in glucose and lipid metabolism lead to the production of excess aldehydes including glyoxal and methylglyoxal. These aldehydes react non-enzymatically with free amino and sulfhydryl groups of amino acids of proteins to form advanced glycation end products. Advanced glycation end-products act directly, as well as via receptors to alter the function of many intra- and extracellular proteins including antioxidant and metabolic enzymes, calcium channels, lipoproteins, and transcriptional and structural proteins. This results in endothelial dysfunction, inflammation and oxidative stress. All these changes are characteristic of hypertension and diabetes [31].

The accelerated non-enzymatic glycation of proteins may be a reason for formation of new epitopes. Advanced glycation of protein causes their immunogenicity. The evidence that AGEs have antigenic properties has led to a hypothesis that the AGE structure found in vivo may exert an autoimmune response [32]. These new antigenic determinants may be more antigenic. It is possible that some of AGE-EDP detected by us form specific antibodies immune complexes. These complexes may form con- sequently to the association of AGE modified elastin and antibodies against AGE-elastin epitopes. The AGE-elastin may becomes substrate for formation of both insoluble IC (it formed at a tissue level) or soluble, circulating immune complexes (CIC) in the serum. For instance, excessively glycated vascular matrix may be an ideal substrate for association with anti-AGE-EDP antibodies. Large insoluble ICs may be formed in this way. It is known that the deposition of immunoglobulins and AGE accumulation are more pronounced in the glomeruli of diabetic kidney than in non-diabetic individuals. The AGE-EDP antibody and ICs may accumulate in the glomeruli, compromising the glomerular basal membrane integrity and contributing to the microvascular complications. Soluble CIC may be formed in the excess of AGE-EDP. CIC generally have a tendency to absorb on erythrocytes, and can also to be bound via other non-specific reactions. Erythrocyte binding of IC is a normal event and pathway of IC delivery to phagocytic cells [33]. Thus, the production of AGE-EDP, anti-AGE-EDP antibodies and AGE-EDP ICs may play a very important role in the onset and progression of the late complications of diabetes. Interactions of AGE autoantibodies with AGE as a continuously produced antigen result in the formation of AGE-immune complexes that may play a role in the atherogenic process [29]. The development of microvascular disease is associated with a high percentage of $\mathrm{HbA}_{1 \mathrm{c}}$. In this study, AGE-EDP of diabetic group 1 correlated with $\mathrm{HbA}_{1 \mathrm{c}}$. $\mathrm{HbA}_{1 \mathrm{c}}$ is a classical example for AGE. These results support the hypothesis for accelerated non-enzymatic glycation.

In conclusion, there is increasing evidence of the association between AGE-EDP and vascular lesions in diabetics with arterial hypertension. Increased levels of serum glycated elastin may be involved in the etiology of micro- and macrovascular complications. The measurement of non-invasive markers of elastin glycation may be useful in monitoring development of vascular wall alterations and therapeutic interventions. Further studies are necessary to elucidate the relationship between the serum level of AGEEDP and vascular complications.

\section{The authors declare no conflict of interest.}

\section{References}

1. Brownlee M (2005): The pathobiology of diabetic complications: a unifying mechanism. Diabetes 54: 1615-1625.

2. Brownlee M (2009): Diabetic complications: pathobiology of hyperglycemic damage and potential impact on treatment. Intertratiol expert workshop. Rome, Italy 2008: 1-8.

3. Baumann M (2012): Role of advanced glycation end products in hypertension and cardiovascular risk: human studies. J Am Soc Hypertens 6: 427-435. DOI: 10.1016/j.jash.2012.10.005. 
4. McNulty M, Mahmud A, Feely J (2007): Advanced glycation end-products and arterial stiffness in hypertension. Am J Hypertens 20: 242-247.

5. Hartog JW, van de Wal RM, Schalkwijk CG, et al. (2010): Advanced glycation end-products, anti-hypertensive treatment and diastolic function in patients with hypertension and diastolic dysfunction. Eur J Heart Fail 12: 397-403. DOI: 10.1093/eurjhf/hfq001.

6. Hayase F, Nagaraj RH, Miyata S, et al. (1989): Aging of proteins: immunological detection of a glucose-derived pyrrole formed during Maillard reaction in vivo. J Biol Chem 263: 3758-3764.

7. Makita Z, Vlassara H, Cerami A, Bucala R (1992): Immunochemical detection of advanced glycosylation end products in vivo. J Biol Chem 267: 5133-5138.

8. Brownlee M, Cerami A, Vlassara H (1988): Advanced glycation end products in tissue and the biochemical basis of diabetic complications. N Engl J Med 318: 1315-1321.

9. Makino H, Shikata K, Hironaka K (1995): Ultrastructure of nonenzymatically glycated mesangial matrix in diabetic nephropathy. Kidney Int 48: 517-526.

10. Brownlee M (1994): Glycation and diabetic complications. Diabetes 43: 836-841.

11. Hammes HP, Weiss A, Hess S, et al. (1996): Modification of vitronectin by advanced glycation alters functional properties in vitro and in diabetic retina. Lab Invest 75: 325-338.

12. Vlassara H, Bucala R, Striker L (1994): Pathogenic effects of advanced glycosylation: biochemical, biologic, and clinical implications for diabetes and aging. Lab Invest 70: 138-151.

13. Murata T, Nagai R, Ishibashi T, et al. (1997): The relationship between accumulation of advanced glycation end products and expression of vascular endothelial growth factor in human diabetic retinas. Diabetologia 40: 764-769.

14. Horiuchi S, Araki N, Morino Y (1991): Immunological approach to characterize advanced glycation end products of the Maillard reaction. Evidence for the presence of a common structure. J Biol Chem 266: 7329-7332.

15. Rosenbloom J, Abrams WR, Mecham R (1993): Extracellular matrix 4: the elastic fiber. FASEB J 7: 1208-1218.

16. Baydanoff S, Nicoloff G, Alexiev C (1987): Age-related changes in the level of elastin-derived peptides in serum from normal and atherosclerotic human subjects. Atherosclerosis 66: $163-168$.

17. Frette C, Wei SM, Neukirch F, et al. (1992): Relation of serum elastin peptide concentration to age, FEV1, smoking habits, and protease inhibitor phenotype: an epidemiological study in working men. Thorax 47: 937-942.

18. Baydanoff S, Nicoloff G, Alexiev Ch (1995): The possible role of the immune system in elastin turnover under physiological conditions. Cor et Vasa 37: 253-255.

19. Baydanoff S, Nicoloff G, Alexiev C (1987): Age-related changes in anti-elastin antibodies in serum from normal and atherosclerotic human subjects. Atherosclerosis 63: 267-271.

20. Fulop T, Wei S, Robert L, Jacob MP (1990): Determination of elastin peptides in normal and atherosclerotic human sera by ELISA. Clin Physiol Biochem 8: 273-278.

21. Colburn K, Kelly G, Malto M, Sandberg L, Boucek R (1992): Serum anti-tropo: anti-alpha-elastin antibody ratio assessing elastin turnover in scleroderma. Clin Rheum 11: 206-210.

22. Labat-Robert J, Robert L (1988): Aging of the extracellular matrix and its pathology. Exp Gerontol 23: 5-18.

23. UKPDS (United Kingdom Prospective Diabetes Study Group) (1998): Intensive blood glucose control with sulphonylureas or insulin and risk of complications in patients with type 2 diabetes mellitus. Lancet 352: 837-853.

24. Bouissou H, Pieraggi MT, Julian M (1985): Aging of elastic tissue in skin and arteries. In: Robert L, Murata K, Nagai Y (eds.). Degenerative diseases of connective tissue and aging. Kodansha, Tokyo: 203-216.

25. Robert C, Lesty C, Robert AM (1988): Ageing of the skin: study of elastic fiber network. Modifications by computerized image analysis. Gerontology 34: 291-296.

26. Cox CS (1991): Roles of Maillard reactions in diseases. HMSO, London.

27. Stone PJ (2000): Potential use of collagen and elastin degradation markers for monitoring liver fibrosis in schistosomiasis. Acta Trop 77: 97-99.

28. Robert L, Jacob MP, Fülop T, et al. (1989): Elastonectin and the elastin receptor. Pathol Biol (Paris) 37: 736-741.

29. Kennedy L (1992): Glycation of immunoglobulins and serum proteins. In International Textbook of Diabetes Mellitus. $2^{\text {nd }}$ ed. Alberti KGMM, de Fronzo RA, Keen H, Zimmet P (eds.). John Wiley, Chichester: 985-1007.

30. Singh R, Barden A, Mori T, Beilin L (2001): Advanced glycation end-products: a review. Diabetologia 44: 129-146.

31. Vasdev S, Gill V, Singal P (2007): Role of advanced glycation end products in hypertension and atherosclerosis: therapeutic implications. Cell Biochem Biophys 49: 48-63.

32. Turk Z, Sesto M, Scodlar J, et al. (2003): Products of advanced glycation in patients with type 2 diabetes and vascular disease. Ann Clin Biochem 40: 552-559.

33. Medof ME, Oger JJ (1982): Competitions for immune complexes by red cells in human blood. J Clin Lab Immunol 7: 7-13. 